\title{
Families in Trouble: Bridging the Gaps Among Child, Adult, and Couple Functioning
}

\author{
Nina Heinrichs $\cdot$ Ronald J. Prinz
}

Published online: 15 February 2012

(C) Springer Science+Business Media, LLC 2012

This special issue grew out of the efforts of an expert work group at a Klaus-Grawe Think-Tank Meeting convened by the Klaus-Grawe Foundation October 2010 in Switzerland. The meeting focused on critical aspects of children, adults, and couples in relation to family adjustment, mental health, and well-being.

Psychological distress is a frequent experience for many children and adults who are living in a family system, even though such distress does not necessarily lead to a diagnosis implicating above-threshold psychopathology. Much research has focused on psychopathology in the individual. This includes individual adults as well as individual children and adolescents. In fact, this orientation toward the individual in clinical research and services is also supported by a system of clinical training that requires that clinical decisionmaking focus on either adults or children. Moreover, within the adult domain, clinicians when building their competencies to deliver services might have to choose whether to concentrate on individuals or on couples. These separations are at times unfortunate, as illustrated by two brief case examples.

In the first example, imagine that you are a child psychologist providing services to a mother who presents one

NIH T32 Behavioral-Biomedical Interface Program:

http://www.sc.edu/bbip/.

N. Heinrichs $(\square)$

Fak. Psychologie und Sportwissenschaft, Universität Bielefeld, 33501 Bielefeld, Germany

e-mail: nina.heinrichs@uni-bielefeld.de

\section{R. J. Prinz $(\bowtie)$}

Parenting and Family Research Center, Psychology Department,

University of South Carolina, Columbia, SC 29208, USA

e-mail: prinz@sc.edu

URL: http://www.psych.sc.edu/facdocs/prinz.html of her two children for assessment and potential treatment. The primary complaint refers to the child's behavior, conceptualized as oppositional-defiant with some symptoms of conduct disorder. In assessing the child and the family, you become aware of the fact that the parents fight with each other, with the most frequent topic of conflict being the child's behavior problems. The mother laments that her husband has withdrawn from the family, leaving her to deal with the child problem on her own. She reports symptoms of depression, which at times fulfill criteria for a major depressive episode. She has thought about seeking individual treatment for her low self-esteem and feelings of inadequacy. The child is likely going to be the focus of the intervention because the mother presented the child's problems as central to her concerns. However, the mother might also receive individual treatment for depression or seek couple counseling. Each intervention might be delivered by a different treatment provider although the dynamics of the problems seem to be interrelated.

In a second example, a couple sees a clinician who is in private practice. The couple reports about a generally distressed relationship in which both partners often experience overt and covert conflicts surrounding a number of topics (such as financial expenses, chores, role distribution, and intimacy). During the assessment and evaluation, the woman discloses depressive symptoms, while the man indicates alcohol abuse. The couple has two children, both under the age of 18 . The younger child is quite anxious in social situations, and both children on occasion have witnessed couple conflicts. In cases like this, some of this information might not even be uncovered because the effects of other members of the family on the individual patient (and vice versa) might not be considered explicitly during assessment and case conceptualization. 
There are a number of areas in which this interrelatedness of individual and relational problems occurs. For example, child maltreatment primarily occurs within the family and is strongly related to parental mental health (Sidebotham and Golding 2001). Child maltreatment can have severe consequences for child development and may result in different mental disorders (multi-finality), each of which in turn requires treatment. Treatments for children exposed to chronic child maltreatment are currently promising, but limited in their efficacy. As such, some of these consequences will likely accompany the individual lifelong and overshadow future relationships.

Another area is adult depression. Depressive disorders show a female preponderance and are often related to couple functioning (Whisman and Baucom 2012, this issue). Furthermore, children tend to live more often with mothers making them more vulnerable to being exposed to a depressed parent. Nevertheless, very little research has focused on family systems (and subsystems) and how these systems interrelate in terms of psychopathology.

The Think-Tank Meeting working group considered these and related issues in their discussions and formulations. By way of background, the Klaus-Grawe Foundation was founded in Zurich in 2005 to promote excellent, interdisciplinary, and innovative research in clinical psychology and psychotherapy and their connected disciplines, with the goal of improving prevention and treatment of psychological problems and disorders. The Think-Tank Meetings represent one of several types of activities hosted by the Klaus-Grawe Foundation that are intended to serve specific purposes. The purpose of the aforementioned meeting was to integrate the latest research findings in basic research, intervention, and prevention on the interrelatedness of child, couple, and adult individual functioning, and to help the research and clinical community become aware of these (sub)system relations. Research in the field of child and adult psychopathology has continued to progress at an impressive rate, both in terms of (a) understanding basic forms of psychopathology themselves and (b) developing and evaluating effective prevention and intervention efforts to alleviate these disorders. However, research has progressed in a way that has left the field somewhat splintered. That is, various investigators and clinicians typically focus upon specific subsystems within the broader family system, for example, the relationship between how an adult's maladaptive behaviors influence a child's functioning, or how having a child with behavior problems can affect the couple's relationship. Thus, research and interventions on children and adults have not been well integrated into a broader family perspective.

The focus of the 2010 Think-Tank Meeting was, therefore, to bring together leading researchers and clinicians who explore different subsystems of the family (child, adult, couple, and family researchers and clinicians) to share their current research efforts and interventions. The intent was to provide a forum that might lead to an integrated perspective on how various family subsystems such as parent-child interactions affect other subsystems such as the couple or the family more broadly. It is essential that if we are to provide broad-based, empirically derived, thoughtful clinical interventions for the field, an integrated perspective on individual, family subsystem, and holistic family functioning be taken into account. This special issue of Clinical Child and Family Psychology Review presents much of the work informing, and emanating from, the working group's efforts.

The articles in this special issue review the literature related to each lead author's primary area of expertise. The first article begins with a review of the interrelatedness of couple and individual adult functioning by Whisman and Baucom. The focus is, therefore, on the couple system and how it affects an individual (and vice versa). The next manuscripts then focus on couples as parents and parentchild subsystems with Cummings and Schatz targeting the issue of how couple conflicts may impact children, followed by Majdandzic and colleagues who investigate the concept of co-parenting in families. Finally, Feinberg, Solmeyer, and McHale focus on the neglected topic of sibling relationships within the family context. From a family generational perspective, the article by Jouriles and colleagues investigates the potential consequences of the intergenerational transmission of exposure to violence as it relates to intimate teen relationships. Rapee next reviews how anxiety disorders in adults and children may be transmitted via family factors, while Ollendick and Benoit conclude the special issue with a model for how social anxiety might emerge in young children within a family system.

Some of the overarching conclusions of this special issue relate to the idea that there are overarching or generic principles of relationships, which apply not only to couple relationships, but also to parent-child relationships and sibling relationships. Among these are the insight (1) that conflicts in relationships are likely beneficial to all relationships when resolved constructively, (2) that all individuals (adults as well as children) have different roles and tasks within and across subsystems, (3) that positive reinforcement, punishment, social skills, and reciprocity are core features of all relationship processes, (4) that a lack of family involvement in evidence-based treatments does not indicate irrelevance of family processes in psychotherapeutic change, and (5) that intervention principles for couple relationships may be expanded to other subsystems involving relationships (e.g., integrated behavioral couple therapy may be expanded and applied to co-parenting conflicts).

To our further knowledge about these potential insights, it is important for researchers to accumulate knowledge 
about these subsystems and relationships in their specific areas of expertise. Clinical work with couples should not fail to assess children's well-being before and after a couples intervention. Clinical work with adults needs to include inquiries about couple relationships (where present) and the children who are part of that relationship. And finally, clinical work with children should consider parents' relationships as couples and parental functioning as individuals.

In sum, the articles in this special issue are truly exceptional in that they venture into new frontiers and allow a glimpse at what might be possible if we integrate such research results into common models and an empirically based set of family interventions.
Acknowledgments Scientific organizers for the Think-Tank Meeting included Don Baucom, Kurt Hahlweg, Mariann Grawe-Gerber, and Nina Heinrichs. We are very grateful to the Klaus-Grawe Foundation, which enabled this Think-Tank Meeting and the articles emanating from the initiative.

\section{References}

Sidebotham, P., \& Golding, J. (2001). Child maltreatment in the "Children of the Nineties": A longitudinal study of parental risk factors. Child Abuse and Neglect, 25, 1177-1200.

Whisman, M.A., \& Baucom, D.H. (2012). Intimate relationships and psychopathology. Clinical Child and Family Psychology Review, 15 (this issue). 